\title{
Making waves: what drives coronary perfusion in the failing heart?
}

\author{
Authors: Sadman Chowdhury, Natalia Briceno, Matthew Ryan, Haseeb Rahman, Simone Rivolo, Jack Lee and \\ Divaka Perera
}

\section{Introduction}

Coronary flow reserve (CFR) is diminished in patients with left ventricular (LV) systolic dysfunction (LVSD) but it is unclear whether this reflects primary microvascular dysfunction or altered autoregulation.

\section{Methods}

Patients with LVSD undergoing elective percutaneous coronary intervention (PCI) were included and those in cardiogenic shock or acute ST-elevation myocardial infarction (STEMI) were excluded. LV pressure-volume loops and coronary flow velocity and pressure measurements were taken following PCI, at rest and during hyperaemia, to calculate CFR and pressure-volume area (PVA = LV stroke work + potential energy (PE)), a measure of myocardial oxygen demand. Coronary wave intensity analysis and wave separation were performed to quantify accelerating and decelerating wave energies.

\section{Results and discussion}

Twelve patients ( $70 \pm 12$ years, LV ejection fraction (LVEF) $27.3 \pm$ $7.8 \%$, post-PCI fractional flow reserve $0.90 \pm 0.12$ ) were enrolled. CFR was $1.6 \pm 0.5$ (resting flow $24.5 \pm 14.4 \mathrm{~cm} / \mathrm{s}$ vs hyperaemic flow $31.4 \pm 19.1 \mathrm{~cm} / \mathrm{s} ; \mathrm{p}=0.005)$; with a positive correlation observed with LVEF $\left(r^{2}=0.56, p=0.020\right)$. Patients with lower LVEF had a higher PVA $\left(r^{2}=0.52, p=0.008\right)$, driven predominantly through $P E$ $\left(r^{2}=0.68, p=0.001\right)$. The magnitude of PE correlated negatively with resting microvascular resistance $\left(r^{2}=0.44, p=0.018\right)$ but not with minimal (hyperaemic) microvascular resistance. Accelerating wave energies were greater in patients with higher PE (forward compression wave $r^{2}=0.56, p=0.005$; backward expansion wave $r^{2}=0.54, p=0.007$ ) (Fig 1). Consequently, patients with higher $P E$ had a greater resting coronary blood flow velocity $\left(r^{2}=0.43\right.$, $p=0.02)$ with reduced CFR $\left(r^{2}=0.54, p=0.025\right)$.

\section{Conclusions}

Reduced CFR in LVSD reflects exhausted autoregulation due to raised PE rather than elevated minimal microvascular tone.

Authors: St Thomas' Hospital, London, UK

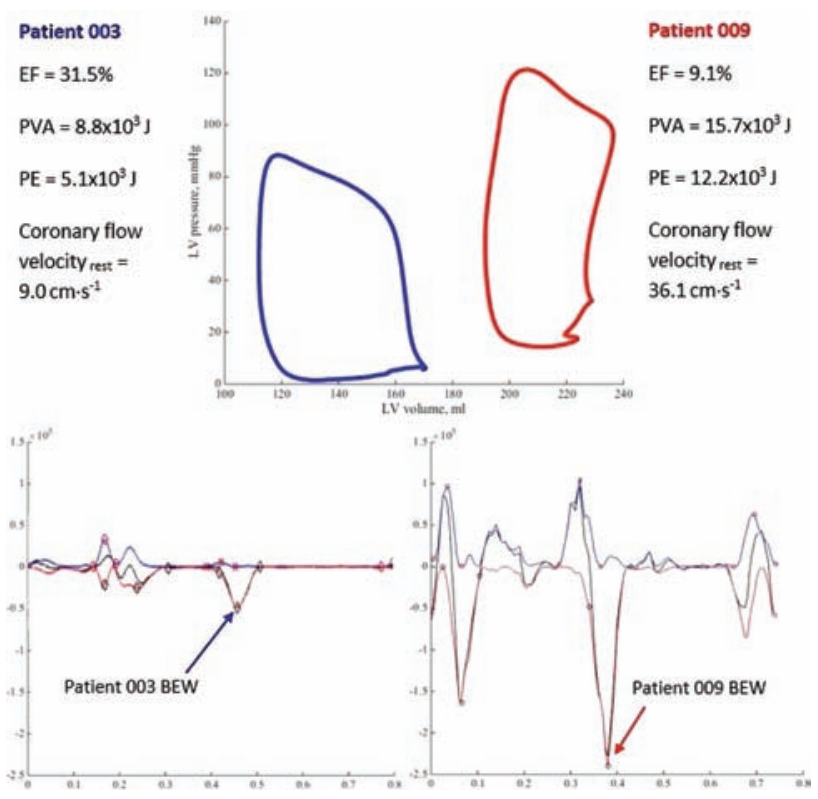

Fig 1. Pressure-volume loops and coronary wave profiles are presented for two patients with better (patient 003) and worse (patient 009) LV ejection fraction.

Strategies to reduce PE, such as mechanical unloading, may improve CFR and protect against ischaemia during high-risk PCI.

\section{Conflict of interest statement}

None declared.

\section{References}

1 Asanoi H, Sasayama S, Kameyama T. Ventriculoarterial coupling in normal and failing heart in humans. Circ Res 1989;65:483-93.

2 Davies JE, Whinnett ZI, Francis DP et al. Evidence of a dominant backward-propagating "suction" wave responsible for diastolic coronary filling in humans, attenuated in left ventricular hypertrophy. Circulation 2006:113:1768-78.

3 Pereira VFA, De Carvalho Frimm C, Rodrigues ACT et al. Coronary flow velocity reserve in hypertensive patients with left ventricular systolic dysfunction. Clin Cardiol 2002;25:95-102. 